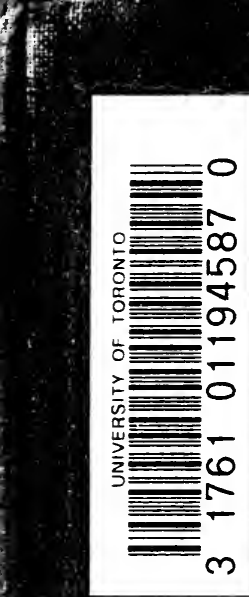

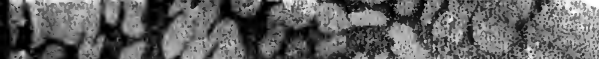

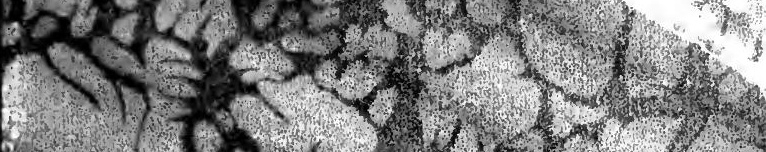

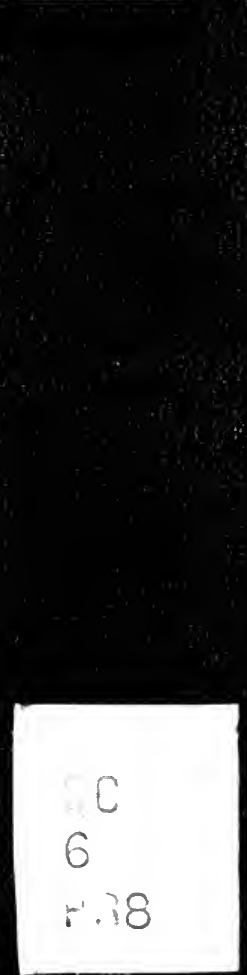

*. 1 , $\triangle 5$

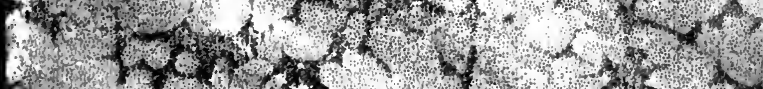
$10 x^{2}=$

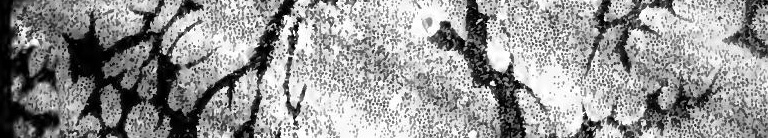
$\operatorname{ran}\left(1,2,4 x^{2}\right.$ $0^{2}+2$, als are 1200

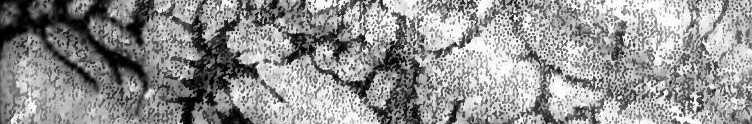

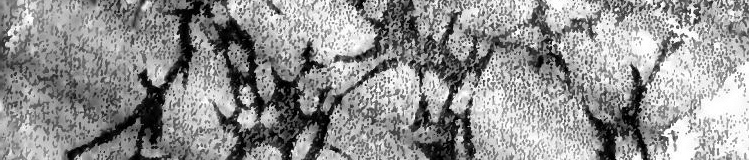
xyor if $x$ 





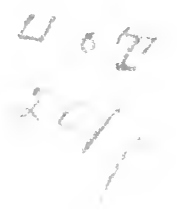

L. A

\section{THÉORIE DE LA RELATIVITÉ}

ET SES APPLICATIONS

A L'ASTRONOMIE 



\section{Astron \\ 75}

LA

\section{THEORIE DE LA RELATIVITÉ}

ET SES APPLICATIONS

1 L'ASTRONOMIE

PAR

\section{M. Émile PICARD,}

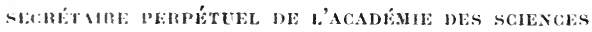

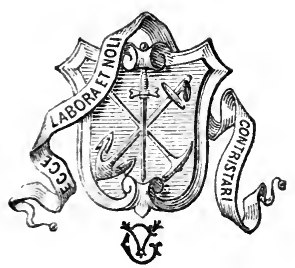

PARIS

GAUTIIIER-VILLARS ET C $\mathrm{C}^{\text {ie }}$, EDITEURS

libraires du bureau des longitudes, de l'école polytechatiqe

55, Quai des Grands-Augustins, 55

192.2 


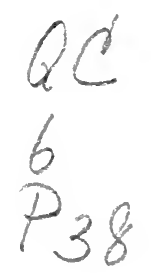

Tous droits de traluction, de reproduction et dadaptation réserve pour lous pays. 


\section{1 \\ TIIEORIE DE LA RELATIVITÉ}

IE SES IPILICITIONS;

\section{A L'ASTRONOMIE ${ }^{(1)}$}

1. On parle beaucoup aujourd'hui de la relativite, et le grand public, qui ne se préoccupe guère habitucllement des théories phrysiques, s’est intéressé à celte doctrine en raison surtout des propositions d'allure paradoxale qu'on y rencontre. II y a vu principalement une modiliration dans les idées fondamentales de l'humaniti relatives à l'espace et au temps. Cependant les questions philosophiques que soulèvent la nature du temps el relle de l'espaee ne datent pas d'hier. Pour Aristote "Le temps est le nombre relatif" au mouvement, lorsque l'on ronsitlère celui-ci comme présentant une partie qui précède et une partie qui suit ", ce que, dans son histoire des doctrines cosmogoniques, Duhem paraphrase sous la forme suivante: Le temps est ce qui permet d'énumérer les états pris pall une chose en monrement en les rangeant dans

(') Cet article a été écrit pour l'Annuaire du Bureau dess Longitudes de 1922 . 
l'ordre de succession. Ainsi, pour le Stagyrite, les notuons de temps el de mouvement sont indissolublement lices; il dil d'ailleurs explicitemént : "Nous mesurons le mourement à l'aide du temps et le temps it l'aide du mouvement ».

2. Une question se pose de suite : le temps n'est-il qu'une idre conçue par notre esprit, ou bien a-t-il me réalite iudfendante de retesprit? Pour Aristole. la seconde alternalive est a adopter. Il admel que. dans des choses réclement existantes, il y a un caractère indépendant de toute intelligence capable de compter; cest ce quil appelle le nombre nombrable

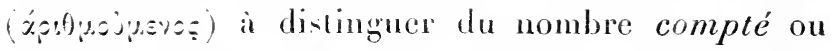

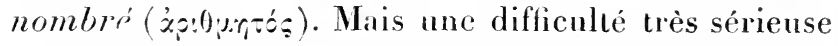
se présente, et qui a posi dans ces temps lointains un problème de relalivilí. P'uisque tout mouvement peut nous donner la notion du temps, la considération de mouvements differents ne nous donnerat-clle pas des temps différents? Aristote répond par la négative. Il roit pouvoir élablir que. quel que soil le mouvement, on aboutira toujours à délinir le même temps, mais sa démonstration ne fut pas, semble-t-il, unanimement admise. Il ne faut pas d'ailleurs oublier que pour les péripatéticiens les substances rouées à la génération et à la corruption sont seules sommises au temps, les ètres qui durent toujours n'étant pas dans le temps; re que reprendra plus lard la doctrine catholique en distinguant le temps et l'éternité. 
:3. Les néoplatoniciens distinguaient deus tomps. Suivant eux, il y a en dehors du temps physique un autre temps, le temps primordial qui est la canse du fremier: au sujet du temps physique, cerlain parlaient du temps d. la lune, de celui du soleil et des planètes. On croirail presque entendre les rolar livistes modernes parler du temps local.

Les discussions commencées dans l'antiquiti su: la nature du temps n'ont jamais cessé durant le moyen ìn et les temps moderne, at les philosophes disserteront sans doute toujours sur son essence. On sait que pour Kant le tcmps el l'espace sont des formes a priori de notre sensibilité, formes a travers lesquelles noum royons nécessairement les choses, el cette opinion sest tronvée ruinée. au moins en ce qui concerne lespace, par l'étude des gómétries non euclirliennes. Plus récemment, une distinction essentielle a fle faite par M. Bergson entre la durée réelle, éest-á-dire la durée vécue que la conscience perçoit, et le tempo paramère qui figure dans nos formules.

1. En présence diopinions si diverses sur le trinps. on est tenté de dire avec sain lagustin: "Quest-ce done que le temps? Si nul ne me le demande, jol. sais: si je cherehe à liexpliquer quand on me l. demande, je ne le sais pas." Ne pouvant délinir h. temps, il faut se borner a le nombrer, pour parler comme les péripatéticiens, éest-à-dire à le mesurer. l'est done reprendre le probleme des temps différeculs, 
un instant souleré dans l'antiquité. Cée préambule u'aura peut-ètre pas été inutile, car, il y a, je crois, intèrèt à rappeler que critainrs difficultés ne datent pas d'hier.

Ceci dit; je voudrai- essayer de tracer une esquisse de la theorie moderne de la relativité, en vued'indiquer les applications à l'astronomic. Je le ferai en narrateur impartial, si jose dire, n'arant pas encore une opinion sur la place que l'avenir réservera à l'édifice si séduisant f)ar certains còtés construit par Einstein, et me demanalant si c'est un progris que de chercher à ramener la physique à la géonétrir, mais plein d'admiration pour l'effort accompli dans cettc audacieuse tentative.

La théorie de la relativité est très abstraite, et c’est, a mon sens, une entreprise vaine que de vouloir l'exposer avec quelque précision sans employer les symboles mathématiques. Comme on l'a dit, il y a des cas où il est plus facile d'apprendre les mathématiques que de s'en passer. J'ai cherché sculement à indiquer ici les idées essentiellr's. sans entrer dans le détail de aalculs souvent trés longs; d'ailleurs la première chéorie de la relativitŕ (relativité restreinte d'Einstein) n'exige que les éléments des mathématiques.

כ. Dans la vie courante, l'homme n’éprouve pas la moindre difficulté en ce qui concerne l'espace et le remps. Il croit avoir une conception très nette d'un 
espace absolu a traver lequel se meuvent les coeps. Cir espace est celui sur lequel raisonnent les géomitres. Quant au temps, il s'écoule d'une manière. régulière et continue, entièrement indépendant de l'espace et indifférent aux phénomènes physiques. A la vérité, pour qui réfléchil quolque peu, la définition de l'unité de temps n'est pas sans présenter de sérieuses difficultés, exrogeant divers postulats sur l'égalité de durées de deux phrinomenes regardés eomme identiques; la difficulti an alténuée toutefois par le fait expérimental que plusicurs phénomènes de nature différcute peurent roncourir d'une manicre concordante à lidée d'égalité de deus temps.

C'est en partant de l'espace et du temps absolus, que s'est peu à peu édifiéc la physique mollerue. D’après les principes mèmes qui sont à la base le notre Dynamique, aucune expérience mécanique. à laquelle sont étrangers les corps extéricurs, effectuée dans un système animé par rapport à l'espace absolez d'un mourement de translation rectiligne et uniforme, ne permct à un observateur entrainé avee ce sistime de mettre le mouvement de colui-ci en évidence; c'rse un principe de relativité. On peut aussi, an lieu de considérer le mouvement par rapport à l'espace absolu, envisager le mourement d'un système par rapport à un autre, par exemple le mouvement de translation de la Terre par rapport au Soleil; aucune difficulté n'est apparue, lant qu'il ne s'est agi que de: phénomènes mécaniques. 
6. Dès le temps d' Irign" at de Fresnel, la mème question se posa pour les phénomènes lumineux. I! résulta de leurs experiences, et de celles des phrsiciens qui les ont snivis. que les lois de la réfraclion et de la róflexion de la lumire ne se ressentent en rien do monvement de translation de la Terre; mais ancune contradiction n'apparut encore à ce sujet. arer les théories admises. Il n'en fut pas de mème d'une expérience, anjourl'hui célèbre, imaginée en เ88; par lo physicien amíricain Michelson, ct trop sourent décrite pour que nous y revenions.

Si l'on armet que la loi habiluelle de composiliom des vitesses de la cinémalique sapplique aux phénomèn lumineax, une contraliction apparait arec le risultat de l'expérienee de Vichelson, un déplacement de franges d'interférence próvu par la théorie classique ue se produisant par. C"est en analysant cette expéricnce qu'Einstein fut conduit, en rgoj. dans son

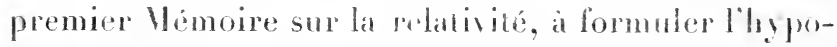
Whèse suivante: la vitessc de la lamière, pour un olsersateur placé dans "un système animé d'un mouvement de translation rectiligne et uniforme, rst une constante unirerselle $c$, indépendante de l'état de mouvement dusystème. Avee ce postulat se trouvait expliqué le fail que l'expérience de Vichelson ne décelait pas le mouvement de la Terre par rapport à l'éther regardé comme immobile, et lon pouvait poser le principe de la relativité pour les phénomènes lumineux dans les mêmes conditions que pour les phŕnomènes mécaniques. 
Ila vérité, certains doutes ont été émis sur l'interprétation de l'expérience de Michclson, le calcul fait a ce sujet prètant à quelques objections; mais ces doutes ne paraissent pas partagés par la majorité des physiciens, et nous admettrons liv principe d'Einstein de la constance de la vitesse de la lumière, d'où va sa tirer toute la théorie de la relativité restreinte (1).

\section{1.}

7. Arant d'aller plus loin, il abl néeessaire de préciser plusieurs points. Jous avons parlé de temps et de longueur. Cula n’a de sens que si les nherervateurs plates sur différents systimes savent mesurer ces grandeurs. Nous arons, par hypothese. un système invariable sinime d'un mourcunent de translation unilurme par rapport à un sistime $\sigma$; ee sera, par excmple, une droite glissant diun mowvement uniforme sur une autre.

8. Soit envisagé $S$; toul d'abord, quand dirons-

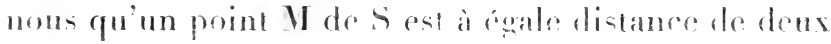

(') Ln point est encore a noter. Dans las thiories classiques relatives à l'éther les équations sont réduitus à la forms: linraire.

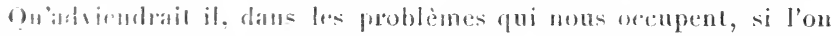

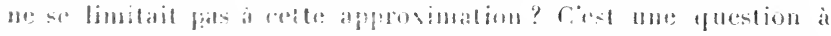

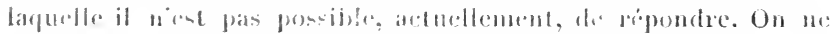

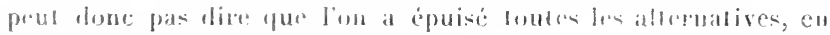
molantanx anciens poinls de vale. 
points $A$ et $B$ de ce mème système? Ce sera quand, les miroirs ayant été placés en ces derniers points, un signal lumineux émis en II reviendra en ce point an mème instant après réflexions en $\mathbf{A}$ et $B$. Il résulte de là qu'un observateur de $\mathrm{S}$ peul faire des graduations lans ce système, sans déplacer des règles.

9. Une autre notion ensentielle est ensuite celle de la simultanéité (dans le système S) de deux événements se passant en $\Lambda$ et B. Cesévénements seront simultanés si des signaux lumineux, partant respectivement de A et B à l'instant où ces événements se produisent, arrivent simultanémen au milieu $\mathrm{M}$ de $\mathrm{AB}$. Le réglage des horloges risulte évidemment de la : deux horloges identiques du sprstème Ś placées en $\Lambda$ et B marcheront laccord si elles marquent la mème heure, quand deux ivénements se produisent simultanément en $\mathrm{A}$ et $\mathrm{B}$. On peut encore dire, relativement à ce réglage, que si un signal, partant de $\perp$ au temps zéro, y revient au temps $\theta$ après réllexion en B, l'horloge en ce dernier point devra marquer $\frac{0}{2}$ quand arrivera le signal lancé par A. Il reste, pour l'observateur entrainć avec S, à faire choix d'une unité de longueur; il prendra pour celte unité une longueur relative à un phénomène physique produit dans le système, par exemple la longueur d'onde d’une radiation délerminée, émise par une source rattachéc au système, et pour unité de temps la périote correspondant à cette onde. 
10. Linsi donc, il y a pour le système $\mathrm{S}$ des unités déterminées et un temps local. Il en est de mème pour le système $\sigma$. Nais comment pourrons-nous comparer les longueurs of les temps de $\mathrm{S}$ et de $\sigma$ ? II ne peut être question de transport dappareils de mesure d'un système à l'autre, ce qui, sans parler des impossibilités, pourait donner lieu à des altérations dont on ne peut rien dire a priori.

Cette comparaison va itre faite en inroquant lo principe d'Einstein de la vitesse constante $c$ de lia lumière, et en tenant compte de la réciprocití der. deux systimes.

Soient $0 x$ la ligne des abscisses dans le système $\sigma$. et $\Omega \mathrm{X}$ la ligne des abseisses dans $\mathrm{S}$; ces droites glissent l'une sur l'autre. L'origine $\Omega$ des coordonnées dans $\mathbf{S}$ a une vitesse $v$ sur $O x$. In point quelconque $M$ a respectivement les coordonnées $x$ et X par rapport aux deux systèmes; $t$ et 'T sont respectivement les temps correspondant i ceux-ci. On veut chercher les apressions de $x$ et $\iota$ en fonction de $\mathrm{X}$ et $\mathrm{T}$, en supposant, comme il est permis far un choix convenable des origines, que $x$ et $t$ s'annulent pour $\mathrm{I}=\mathrm{T}=\mathbf{0}$.

$\mathrm{Si}$ le couple $(x, t)$ correspond à unc onde lumineuse, sa vitesse $\frac{d x}{d t}$ étant égale à $c$, on aura

$$
d x^{2}-c^{2} d 1^{2}=0:
$$

mais, d'après l'hypothèse d'Einstein, cette équation devra entraîner

$$
d^{2}-c^{2} d \top^{2}=0 .
$$


Une de ces équations entraine donc l'autre. Les premiers membres de ees équations ne diffèrent donc. que par un facteur. et la réciprocité (') des deux systimes exige que ce facteur soit égal à l'unité. On a dons:

$$
c^{2} d l^{2}-d x^{2}=r^{2} d T^{2}-d X^{2},
$$

ce qui détermine les expressions cherchées, an ajoutant la condition que la vitesse de $\Omega$ par rapport à $O$ est égale à r. On trouve ainsi

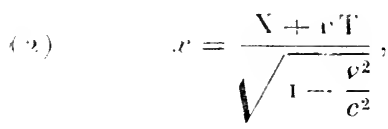

$$
\prime=\frac{\mathrm{T}+\frac{b \mathrm{X}}{c^{2}}}{\sqrt{\mathrm{1}-\frac{c^{2}}{c^{2}}}}
$$

d'où se déduit de suite

(3)

$$
\mathrm{X}=\frac{x-1 \cdot}{\sqrt{1-\frac{v^{2}}{c^{2}}}} .
$$$$
\mathrm{T}=\frac{1-\frac{v x}{c^{2}}}{\sqrt{\mathrm{I}-\frac{c^{2}}{c^{2}}}},
$$

équations auxquelles on adjoindra $y=r, z=Z$, ces deux coordonnées n'ayant pas changé puisque $\mathbf{S}$ a, par rapport à $\sigma$, une translation dans le sens de l'ase des $x$.

Si le sens de la translation uniforme avait été quelconque. on aurait eu une transformation plus

(1) Cette réciprocité s'exprime en écrivant que la longucur, dams le système $\sigma$, d’une longueur un immobile par rapport ì $S$ est igale à la longueur, dans le srstème $S$, l'une longueur un in:mobile par rapport à $\sigma$. 
„énérale, se rattachant ì l’invariance (4), qui généralise (I) :

(i) $c^{2} d t^{2}-d x^{2}-d^{2}-d z^{2}=c^{2} d^{2} \mathrm{~T}^{2}-d \mathrm{X}^{2}-d \mathrm{I}^{2}-d l^{2}$,

et le groupe correspoudant porte le nom de Lorent:, qui l'a systématiquement envisagé.

On roit le rôle joúé par les signaux lumineu.r. rans les mesures de l'espace et du temps, ainsi que le postulat résultant dr linterprération de l'expririence de Michelson. Si l'on n'adopte pas ces point. le vue, la théorie n'a plus de base.

11. Tirons diverses conséquences des équations (2) et (3). Soit une longueur $\mathrm{I}_{1} \mathrm{M}_{2}$ sur $\mathrm{S}$, les abscisses des extrémités $M_{1}$ et $\Pi_{2}$ sur $\Omega$ X élant $\triangle_{1}$ et $X_{2}$. \ $\| \prime \prime$ méme temps $t, \mathbf{M}_{1}$ et $\mathrm{I}_{2}$ occupent sur $\sigma$ les positions $m_{1}$ et $m_{2}$ correspondant aux abscisses $x_{1}$ et $x_{2}$ sur $\mathrm{O} x$. On a, d'après la première des équations (3).

$\therefore$ est-à-dire que

$$
\mathbf{X}_{2}-\mathrm{S}_{1}=\frac{x_{2}-x_{1}}{\sqrt{1-\frac{v^{2}}{c^{2}}}},
$$

$$
\overline{m_{1} m_{2}}=\sqrt{1-\frac{r^{2}}{c^{2}}} \overline{\Pi_{1} \|_{2}} .
$$

Par suite, pour l'observaleur sur $\sigma$, la longueur $\overline{\bar{M}_{1} M_{2}}$ est réduite, et cela d'autant plus que rest plus sriand.

Supposons eneore quin phénomène se passe an $\Omega(\backslash-0)$, et dure un temp- T pour l'observatem. 
sur S. L'observateur sur $\sigma$ lui altribuera la durée plus longue

$$
t=\frac{\mathrm{T}}{\sqrt{1-\frac{r^{2}}{c^{2}}}},
$$

conme il résulte de la seconde des équations (2). Le temps avance donc moins vite pour l'observateur fixe que pour l'observateur mobile. Un homme en mouvement vieillit par suite plus lentement, du moins a l'estime d'un homme au repos.

12. En différentiant les formules (2), et en divisant, on voit que la loi de composition de deux vitesses $"$ etrest donnée par la formule (on pose $\frac{d X}{d T^{1}}=u$ )

$$
\frac{u+r}{1+\frac{u r}{c^{2}}} .
$$

La cinématique de la relativité ess donc différente de la cinématique classique, pour laquelle la loi de composition est $u+r$; les lois ne coüncident que pour $c=\infty$.

Divers phénomènes sont bien d'accord avec le résultat précédent. Citons sculement ce qui concerne la vitesse de la lumière dans un liquide en mouvement. Soient $u$ la vitesse du liquide, $\frac{c}{n}$ la vitesse de la lumière dans le liquide en repos ( $n$ étant l'indice de réfraction). I a vitesse résultante est, d'ap rès la formule précèdente, 
non pas $u+\frac{c}{n}$, mais

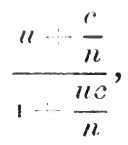

$\because$ est-à-dire approximativement $\frac{c}{n}+u\left(\mathrm{I}-\frac{\mathrm{I}}{n^{2}}\right)$; le - econd terme correspond au transport partiel de l'onde lumineuse proposé par Fresnel, et ce résultat est d'accord avec unc experience célèbre de Fizcau faite en 18 э. . Remarquons d'ailleurs que, dans le raisonnement précédent, on étend à un milieu transparent le postulat de l'isotropie de la vitesse de la lumire admis pour le vide.

13. D'après la théorie de la relativité restreinte, il n'y a pas pour les phénomènes d'échelle absolur de mesure, indépendante des systèmes dans lesquels ils sont observés. En outre, le temps et l'espace jouent des rôles analogues, sinon identiques, dans les équations. Il n'y a donc pas, dans la doctrine de la relativité, d'un côté un espace $(x, y, z)$ et un temps $\iota$, mais un continuum $(x, y, z, t)$ à quatre dimensions. Les phénomènes se passent dans ce continuum que Minkowski appelle l' Unirers. Celte idée a été expriméc par ce géomètre dans une conférence faite en r 908 . trois ans après l'apparition du premier llémoir d'Einstein, sous la forme suivante : "Dès maintenant l'espace indépendant du temps et le temps indépendant 
le l'espace ne sont plus que des ombres vaines; une sorte d'union des deux doit seule subsister encore."

Ln événement est représculé par un point de: l'Lnivers, et il decrit dans ses modifications une ligne I'Univers. L'expression

$$
d s^{2}=c^{2} d^{2} \cdots d x^{2}-1 r^{2}-d z^{2}
$$

ne: ehange pas quand on effectue sur $x, y, z, \iota$ les lransformalions du groupe de Lorentz, et l'intégral.

$$
\int_{-1}^{d i} d s
$$

prise sur une ligne d'Univers entre les événements correspondant à ses extrémités $\mathbf{A}$ et $\mathbf{B}$ est appelér l'intercalle entre ces érénements. Guidé par des analogies avec la théorie des lignes géodésiques des surface est l'ordre d'idés s mattachant au principe de la moindre action, on pose qu'un mobile libre décrit une géodésique de l'Lnivers de Minkowski, et que, par suite, pour la trajectoire de ce mobile, la variation de l'intervalle 'ntre deux événements est égale à zéro, ce qui s'exprime par

$$
\delta \int_{1}^{k} d s=0 .
$$

jótant le: symbole ordinaire lu calcul des variations. Un calcul facile montre qui, ces trajectoires sont des lignes droites parcourues uniformément. Les rayons Lumineux sont des géodésiques de longueur null. $(d s:=0)$. 


\section{III.}

14. Einstein a été peu à peu conduit à unc extension considérable de sa première théorie. Reprenons 1. ds² envisagé plus haul:

$$
d s^{2}=c^{2} d t^{2}-d x^{2}-d y^{2}-d z^{2} .
$$

Si, an lieu des coordonnces $x, y, z, \ell$, now fiaison: unage de coordonnées généralisées $x_{1}, x_{2}, x_{3}, x_{2}$, de telle sorte que $x, y, z, t$ soient des fonctions de $x_{1}$, $r_{2}, x_{3}, x_{4}, d s^{2}$ deviendra une expresision de la forma (i) $\quad d s^{2}=-g_{i} d_{i} d r_{i} d r_{k} \quad\left(s_{i k}=g_{k i}\right)(i, k=\mathbf{1}, 2,3, i)$ les gótant des fonctions de $x_{1}, x_{2}, x_{3}, x_{4}$; on pent. far exemple, supposer que $x_{1}, x_{2}, x_{3}$ sont des coordonnées curvilignes dans l'espace ordinaire et $x_{4}$ une coordonnée relative à une horloge chargée seulcment de définir lordre de succession des événements atutour du point $\left(x_{1}, x_{2}, x_{3}\right)$. I l' nivers envisagé sa lrouve ainsi attachée une cortaine forme $(6)$.

Prenons maintenant la question en sens inverse, cl donnons-nous arbitrairement dix fonctions $g_{i k} d e \cdot x_{1}$, $x_{2}, x_{3}, x_{4}$ et, par suite, la forme qualratique $(6) \mathrm{de}$ différentielles. Un problème se pose d'abord: peut-on de (b) remonter à (j) en prenant pour les $x_{i}$ des fonctions convenables de $x . y, z, \ell$ ? La reponse cost negative : la chose n'est possible que si hres satisfont a ringt relations renfermant les ge el leurs derives particlles jusqu'au second order. Si ars conditions 
sont remplies, on dit que l'Univers correspondant à (6) est euclidien ( '); il en est ainsi, en particulier, quand les $g$ sont des constantes.

Supposons-nous maintenant dans le cas général, où l'Univers donné par (6) n'est pas euclidien. Nous considérons alors en un point déterminé, d'ailleurs quelconque, de cet Univers un Univers euclidien qui lui soit tangent. Voici ee quon entend par là. Le point étanl donné, la forme quadratique (6) est une forme quadratique des $d x_{i}$ à coefficients constants. On peut alors choisir $d x, d y, d z, d t$ fonctions tinéaires et homogènes de $d x_{1}, d x_{2}, d x_{3}, d x_{4}$, de telle sorte que (5) se transforme en (6). Notre Univers initial a étć ainsi transformé en un Univers euclidien, mais seulement dans le voisinage du point envisagé : cot Univers euclidien est l'Univers tangent en ce point.

Dans les univers euclidiens tangents aux divers points, supposons, pour fixer les idées, que les observalcurs prennent pour unité de longueur une certaine

(1) Arec plus de précision, une forme telle que (6) est dite euclidienne quand elle est susceptible d'ètre ramenée à la forme

$$
d \mathbf{S}_{1}^{2}+d \mathbf{S}_{2}^{2}+d \mathbf{S}_{3}^{2}+d \mathbf{X}_{4}^{2},
$$

les $\mathrm{X}$ ćtaut des fonctions des $x$, la transformation étant complexe aussi bien que réelle; ceci correspond à ce qu "Riemann appelait espace plan. Si l'on reste dans le domaine rérl, certains carrés pourront ètre précédés du signe moins; c'est ce qui arrire pour la forme (5), et il peut arrirer alors que toutes les variables nc jouent pas le méme ròle, tel le temps dans cette dernière forme. Aussi faut-il parler d'analngie et non d'identité entre l'espace et le temps. 
longueur d'onde lumineuse et une unité de tempsrelle qu'elle donne à la vitese de la lumière unevaleur $c$. Dans ces conditions, une correspondance. se trouve établic de proche en proche entre les observations faites dans notre Univers et celle des observateurs des Univers euclidiens tangents. C'est là un point fondamental, mais l'application de cette idéc. générale n'est pas sans présenter quelques difficultés, comme on le verra plus loin sur un cas particnlier.

13. On pose en principe que toute loi physiquedoit ètre exprimée par des équations gardant la mème forme, quand on substitue aux coordonnées généralisées des fonctions quelconques de celles-ci, ce qur̈ constitue sous sa forme générale le principe de. relativité. Il en est bien ainsi pour les équations dı mouvement d'un point libre; elles sont obtenues en écrivant que la variation de l'intervalle entre deu. écénements A et B est nulle, c'est-à-dire

$$
\partial \int_{1}^{1} d s=0
$$

et elles gardent manifestement la mème forme invariante par rapport au $d s$ transformé. Les trajectoirecorrespondantes sont dites les géodésiques correspondant au $d s$.

La physique d'un Univers dépend done de son ds C'est ce qui a lait dire que la pliysique se trouveramenée à la géométrie. 


\section{W.}

16. Dans un Lnivers auclidien, on dit qu iil n'y a pas de champ permunent de gravitation. Donc dans un Univers, oì il a un champ gravitationnel, les $g$ ne satisfont pas aux ringl équations dont nous avons parté plus haut. D'autre part, s'il y a, pour un Univers, des relations entre les $g$ et leurs dérivées particlles le caractérisant, les idées d'invariance imposies par le principe de rilatis ite exigent que l'ensemble de ces relations ail me forme invariante quand on fait un changement quelconque de coordonnées. C'est le zran! mérite d'Einstein d'anoir su tronver un systèm. de relations entre les $s$, moins limitatif que les vingt équations relatives a l'espace euclidien, et capables, suivant lui, de caratériser un espace gravitationnel.

Nous ne pourons songer ici a indiquer les diverses hypothèses et les laborieux calculs qui ont conduit à ces relations, se présentant en nombre d'abord égal à dix, mais réduclible à six. Disons seulement qu'Einstein a pris prour point de départ l'hypothèse qu'une transformation consenable de coordonnces est équivalente, dans le voisinage d'un point, à unc force de gravitation; éest lí l'idée directrice qui permet de ne plus parler le force et, en fait, dans cette géométrisation de la physique, l'attraction liest plus une force, mais une propriété de l'espace. Les propriétés de la matière se rattachent à cette 
géométrie à quatre dimensions par des généralisations de l'équation classique de Poisson dans la théorie du potentiel newtonien.

Je n’ai pas cru devoir dan- l'exposition qui préccill. parler de courbure dans l'espace à quatre dimensious. II n'y a lì qu un langage plus ou moins commode, car nou n arons pas l’intuilion din tel espace. Pent-ètre mème a-t-il conduit parfois at des assertions en opposition avec la pure doctrine de la relativité. Ainsi on peut live chez cortains auteurs que la présence de la matière produit une courhure do l'espace, tandis qu il et plus conforme aux idées de séométrisation de dire que l'existence de la matiore "st une conséquence de certaines deformations. Je niti rien dit non plus de la masse, notion qui ne jour pas de role direct dans les applications faites icia l'astromomie; ce qui li concerne doit. pour ètre présonté dans toute son ampleur, èce ratlaché aux formules compliquées de la relativité générale.

17. Les seuls controles experinentaux auxquels a été soumise jusqu'ici en astronomis la théorie de la relativité se rapportent à un (hamp) ponctuel de gravitation provenant d'un point que nous supposeron. placé à l'origine. La recherche du als correspondant renferme beaucoup d'indétermination. Le problèm. peut ètre précisé au moven de diverses hypotheses simplificatrices. Dans la forme la plus usitée, ‘n appelle $t$ la quatrième variable $x_{4}$, et $x_{1}, x_{2}, x_{3}$ repró- 


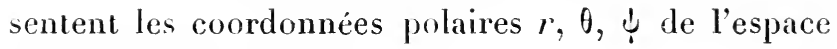
habitucl. On a alors

$$
d s^{2}=\gamma^{2} d l^{2}-\frac{d r^{2}}{\gamma}-r^{2} d 0^{2}-r^{2} \sin ^{2} \theta d \bigcup^{2},
$$

où $\gamma=1-\frac{2 m}{r} ; m$ est une constante introduite dans le courant du calcul.

Le mouvement d'un point libre dans l'espace (8) se détermine facilemeni. L'orbite est approximativement une ellipse, dont l'origine est un foyer. Mais le grand axe de cette ellipse n'est pas fixe, comme dans le cas newtonien du problème des deux corps. Il tourne à chaque tour d'un angle sensiblement égal à

$$
\frac{24 \pi^{3} a^{2}}{c^{2} T^{2}\left(1-e^{2}\right)}
$$

$a, \mathrm{~T}, e$ désignant respectivement le grand axe, le temps de la révolution et l'excentricité; de là se déduit la rotation séculaire.

18. Une application de la formule donnant la rolation séculaire a eu un grand retentissement; c'est cell. qui concerne le mouvement du périhélie de la planètr. Mercure. On sait que, dans la mécanique céleste classique, le mouvement de Mercure est approximativement aussi un mouvement elliptique avec rotation du grand axe, mais celte rotation tient à l'action des autres planètes, particulièrement de Vénus. Or la théorie des perturbations donne seulement pour le périhélie une avance, de $532^{\prime \prime}$ par siècle, tandis que 
lobservation donne une avance de $\check{5}-4^{\prime \prime}$. Il y a donc un résidu d'environ $42^{\prime \prime}$, mais il me semble que ce nombre ne soit pas déterminé arec une grande précision. Malgré des tentatives rariées (changement de l'exposant dans la loi de Newton, aplatissement du Soleil, planète intramercurielle, lumière zodiacale, loi électrodynamique, etc.), on n’a pas réussi à expliquer d'une manière satisfaisante ce résidu ( ').

Einstein s'est demandé ce que donnerait la théorie nouvelle appliquée à Mercure, en ce qui regarde l'action solaire, rien n'étant changé d'ailleurs dans l'action des planètes. Or il est remarquable que, en introduisant dans la formule (9) les nombres relatifs a Mercure ( $\left.{ }^{2}\right)$, on trouve $42^{\prime \prime}, 9$ : cest la différence cherchée. On pourrait presque dire que ce résultat est trop satisfaisant, tant d'influences incomplètement

( $\left.{ }^{1}\right)$ Tout récemment (Comptes reulus 29 aoủ 192I), M. Gaston Bertrand a fait l'intéressante remarque qu'une infinité de lois d'altraction, dépendant à la fois de la distance et de la vitesse, conduisent à la formule ( 9 ), et rendent compte par suite du résidu relatif à Mercure. Telle est en particulier la loi

$$
f \frac{m m}{r^{\prime}}\left(1-\frac{r^{2}}{c^{2}}\right)^{-3}
$$

ne difrérant de la loi de Newton que par le dernier facteur dépendant de la vitesse $v$. Celte loi et eelles visées plus haut ont évidemment un caractère artificiel, et ne se rattachent pas, commc il arrive pour l'application faite par Einstein. à une théorie générale conçue sans souci du eas particulier à expliquer.

(2) L'unité de longueur étant le kilomètre et l'unité de temps le Irois-cent-millième de seconde. 
analysées jusqu ici devant s'exercer dans le voisinage.

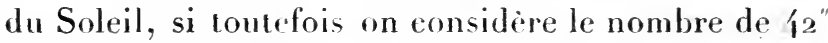
eomme correspondant réllement aux observations.

Pour les autres planctes, sauf peut-ètre Mars, la rotation du périhélie rst tris mal déterminée, a cause de la petitesse de l'excentricité, car la quantité mesurée est le produit de la rotation par l'excentricité; il n'y a donc pas lieu de leur appliquer la théorie précédente. Quant à Mars, il y a entre l'observation et la théorie classique un résidu de $4^{\prime \prime}$, susceptible peut-c̀tre de s'expliquer par l'attraction des astéroüdes circulant entre Mars et Jupiter. Quoi quail en soit, si l'on applique la formule (9), on trouve pour la rotation du grand axe de Mars pendant un sieele le nombre 1",35: le résidu non expliqué de 4 " serait done diminué de ce nombre.

19. Un seconil problime relatif à l'espace gravilationnel (8) appelle l'attention. Quelle est la trajectoire d'un rayon lumineux? Il faut chercher, comme nous l'avons dit, les géorlésiques de longueur nulle. Le rayon lumineux est approsimativement hyperbolique. Si la distance du sommet de celte courbe au centre de gravitation est $R$, on a pour l'angle \% des asymptotes

$$
x=\frac{i m}{R}
$$

et cet angle est sensiblement l'angle de déviation des rayons lumineux. Ceci trouve son application 
dans le cas d'un rayon lumineux renant d'une étoile et rasant le bord du Soleil. Il est naturel d'admettre que le sommet est à la surface du Soleil; R étant alors le rayon solaire et $m$ étant égal à $\left\{\pi^{2} \frac{a^{3}}{c^{2} \mathrm{~T}^{2}}(a \mathrm{ct} \mathrm{T}\right.$ correspondent à la Terre), la valeur de o est égale ¿ $1^{\prime \prime},-5$. Le rayoon lumineux est donc dévié de cet angle.

Les étoiles ne pouvant ètre ohservées près du Soleil, il faut ehercher à profiter d'une éclipse totale de cet astre pour observer cette déviation. Ciest ce qui a été fait avec succès dans l'apédition organisće par l'éminent astronome anglain II. Eddington pour observer l'éclipse de Soleil du sy mai 1010. L'accord avec la théorie semble s’ètre montré satisfatisant. mais il est a souhaiter qu une échipse prochaine confirme ces mesures délicates. On doit désirer ausi que laction de la matière sur la lumière puisse ètre mise en évidence dans des conditions plur facilement observables.

20. Une dernière application de la théorie de la relativité à l'astronomie concerne le déplacement vers le rouge des raies du spectre solaire par rapport a celles des sources terrestres, sous l'action du champ de gravitation provenant du Soleil. Les consilterations faisant prévoir ce déplacement peuvent ètre présentées de la manière suivante:

Plaçons-nous en un point donné $(r, f, j .1)$ de Thivers correspondant ì (8). Diprès ce que nous 
avons dit (\$14), on doit identifier (8) avec le carré $c^{2} d \mathrm{~T}^{2}-d \mathrm{X}^{2}-d \mathrm{Y}^{2}-d \mathrm{Z}^{2}$

de l'élément de l'espace euclidien, où $\mathrm{T}$ désignera le lemps dans l'Univer enclidien tangent. On obtient ainsi immédiatement

$$
r \mathrm{~T}=\left(1-\frac{2 m}{r}\right)^{\frac{1}{2}} d t .
$$

Considérons alors un atome vibrant placé au point considéré. Si $t_{0}$ et $T_{0}$ sont les deux durées des vibralions correspondant respectivement à un point de (8) a la distance $r_{0}$ du Soleil et a l' L nivers tangent, on aura

$$
\mathrm{T}_{0}=\left(1-\frac{2 m}{r_{0}}\right)^{\frac{1}{2}} t_{0}
$$

Or on admel, é qui peul itre contesté (c'est à cetle difficulté que nous faisions allusion à la fin du paragraphe 14), que $\mathrm{T}_{0}$ est fixe; ceci revient à supposer qu'il y a chute libre dans l'C nivers tangent. Par suite, pour deux points situés à des distances $r_{0}$ et $r_{0}^{\prime}$, on a, entre les temps $\iota_{0}$ et $t_{0}^{\prime}$, la relation

$$
\left(1-\frac{2 m}{r_{0}}\right)^{\frac{1}{2}} l_{0}=\left(1-\frac{2 m}{r_{0}^{\prime}}\right)^{\frac{1}{2}} t_{0}^{\prime} .
$$

$\mathrm{Si}$ le second point est sur la 'Terre, on peut regarder $r_{0}^{\prime}$ comme infini, et en se bornant à la première puissance de $r_{n}$, on peut écrire pour un point sur le Soleil

$$
t_{0}=\left(1+\frac{m}{r_{0}}\right) t_{0}^{\prime},
$$

où $r_{0}$ représente le rayon solaire. 
La durée de la vibration $\iota_{0}$ pour l'observateur sur le Soleil est done plus grande que la durée $t_{0}^{\prime}$ pour lobservateur sur la Terre. Il doil done y aroir une dériation vers le rouge. En passant aux nombres, on a

$$
t_{0}=i_{0}, \mathrm{r}, 0000021 \text {. }
$$

On attachait une grande importance à la vérification expérimentale de ce résultal, sans doute parce qu'il paraît tenir à des propriétés intimes de la matière. Mais cette vérification élait difficile, car au déplacement prévu par Einstein peut s'ajouter un déplacement des raies par la pression. sur l'importance duquel on n'est pas fixé. Toutcfois, M. Perot croil avoir établi que pour certaines raies du magnésium l'effet de pression est négligeable, et que l'écart entre les raies du spectre solaire el celles de l'are sous faible pression a la valeur prévue par la théorie d'Einstein. Dautre part, MM. Fabry et Buisson, en examinan! d'anciennes mesures faites par eux ì une époque où l'on ne soupçonnait pas linfluence de la gravitation. ont constaté que leurs résultats étaient en concordance parfaite avec la théorie de la rolativité. Pour les raies du fer qu'ils ont examinées, comme pour celles du magnésium étudiées par M. Perot, la pression de la couche renversante serait négligcable, et l'eflet Einstein serail seul en cause. Mais il est sans doute prudent d'apporter encore quelqur réserve dans cette affirmation. 


\section{V.}

21. Telles sont les applicalions faites jusqüici i l'astronomie des vues dEinstein sur la gravitation. Elles se rapportent loutes les trois, on doit le remarquer, a des phénomines se passant sur le Soleil ou à une distance relativement faible de cet astre. Pour que la physigue s'engage définitivement dans la voic morerte par la théoric de la relativité, il faudra probablement que de nombreuses expériences, d'un caraction posilif. aient dif clfectuées dans les laboratoires. I ne expérience, se rattachant aux questions gui nous occupent, fut laite, il $y$ a une dizaine d'années, par M. Sagnac. Elle présente quelque analogie ave celle de Nichelson, et se passe dans un systeme auquel on donne $u n$ mourement de rotation, mais elle se rapporte anx éléments du premier ordre. Dans cette expérisnere. on olserve un déplacement de franges d'interférence qui met en évidence la rotation. Il est d'ailleurs possible d'expliquer la belle expérience de 11. Sagnac au moyen de la théoric générale de la relativité, comme au moyen des théories classiques.

Terminons par quelques remarques générales. Certains trouvent qu'il y a dans l'établissement de la théoric de la relativité einsteinienne des points obscurs et des hypothèses insuffisamment formulées, et cela est exact. Mais rette rilique touche peu ceux qui 
pensent qu'une throric ne doit pas avoir la prélention de donner des apparences une explication conforme a la ralite, ol que seuls importent les formules finales et leur accord aussi exarl que possible arec un ensemble de lois exprimentales, le but essentiel étant

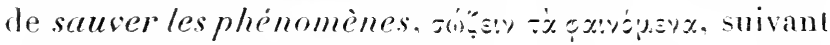
une expression qui remonte a Platon. et la partic esentielle diune theoric chant surtout le moule anaI tique dans lequel ello enferme les choses. I ce poim de vue. on peut dire que re qui constitur une théoric 1. Ia relativite, e'est le $/ s^{2}$ qui lui correspond. Celuici obtenu, on peut faire abstraction de la manière dont (1) y a été conduit.

D'aulres, atlachris anx idées tradiliomnelles, ne pernnent pas facilement leur parti d'une sorte d. rupture aree le sens commun. L'arenir dira dans quelle mesure, si de nouveaur fais exporimentaus leur apportent un appui, les idées nouvelles pourront suncorporer dans a hon sens moyen de l'humanite. où Descartes mettait le fomdement de la certilude, (') qui était pour lui le trait diunion entre notre penséc et le réel. Sans cet accord, il n’y a que scepticismo; rest un écueil que n’ont pas loujours évité les théoricions de la phisinge. 
PARIS. - IMPRIMERIE gattiler-VILlars et $c^{\text {io }}$; 66919 Quai des Grands-Augustins, 55. 




QC

6

P38

P\&ASci

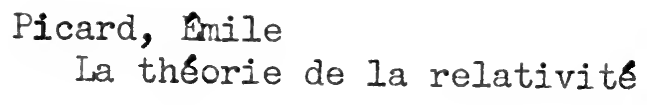

PLEASE DO NOT REMOVE

CARDS OR SLIPS FROM THIS POCKET

UNIVERSITY OF TORONTO LIBRARY 

$-7 \times 2^{25}+10$ rater mon: tont 1 is

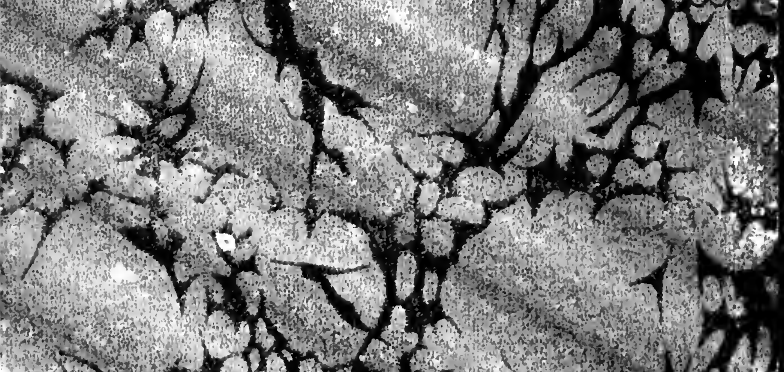

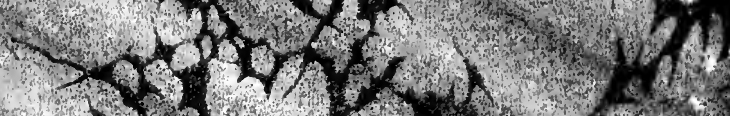

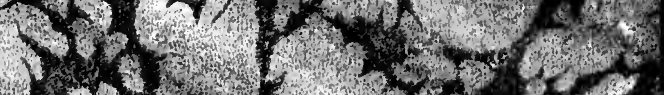

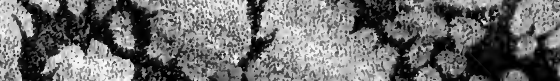

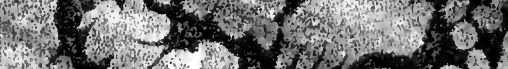

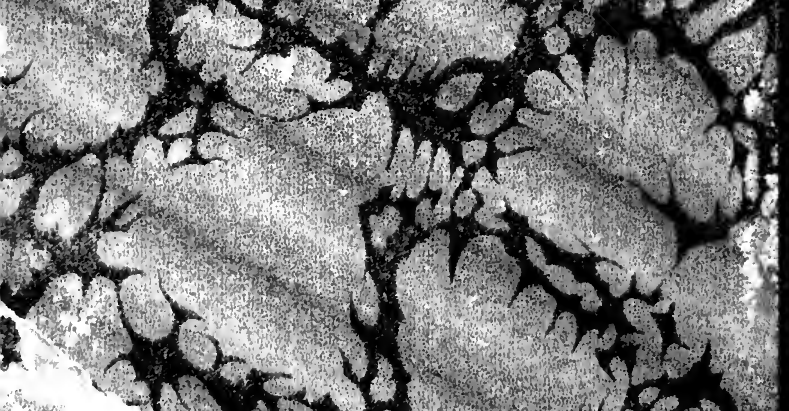

